\title{
Zur Einschätzung von Waldfunktionen durch Vertreter öffentlichen Waldeigentums - ein regionaler Vergleich
}

Die wachsende Umweltbelastung hat in den letzten Jahren den Wald zu einem wichtigen Thema des öffentlichen Interesses gemacht. Die Erörterungen der Waldschadensituation dürften überdies dazu beigetragen haben, die ökologischen Zusammenhänge und die Langfristigkeit der Prozesse in Erinnerung zu rufen. Andererseits mag die gesetzliche Sonderstellung des Landschaftselementes Wald in der Schweiz ausschlaggebend gewesen sein, daß forstliche Themen von Geographen lange wenig beachtet wurden. Der Kulturlandschaftswandel zeigt sich bei denWäldern zwar in der Regel nicht so deutlich und augenfällig wie derjenige in der Agrar-oder gar der Siedlungslandschaft. Es sind indessen von einem integralen Ansatz aus vielfältige Interessenkonflikte mit dem Wald auszumachen, die raumwirksame Folgen nach sich ziehen (vgl. z. B. EWALD 1978). Die nachstehenden Zeilen möchten bei einem Teilaspekt, dem Waldeigentum, ansetzen.

\section{Waldeigentum}

Seit mehr als hundert Jahren sind die Schweizer Wälder einer für das Grundeigentum relativ restriktiven Gesetzgebung unterstellt. Gleichwohl ist eine große Bedeutung der Waldeigentümer als Aktoren forstlicher Veränderungen anzunehmen. Gerade sie gelangen jedoch, verglichen mit dem Forstdienst und der vielgestaltigen Gruppe der Waldbenützer, selten zu Wort. So meinte M. de COULON (1983: 707-708):

"La forêt publique, malgré son importance, est plus ou moins anonyme: elle appartient à tout le monde, donc à personne. Officiellement, quelque 4000 autorités (cantons, communes, bourgeoisies, corporations, etc.) la représentent. De fait, les intérêts de la forêt publique sont défendus par quelques centaines de forestiers: les techniciens veillent au grain, et assurent la permanence. Dans l'arêne politique, hélas, quelques centaines d'électeurs et quémandeurs sont quantité totalement négligeable.» Und er fuhr fort: "Sur le plan politique, la forêt privée est d'ordinaire encore plus inexistante que la forêt publique. Et pourtant elle a près de 200000 propriétaires."

Die Aktivitäten solcher sozialgeographischen Gruppen zu untersuchen, bildet einen wichtigenThemen- bereich forstgeographischer Forschung (WINDHORST 1978: 14).

Drei Viertel der Waldfläche der Schweiz stehen im Eigentum von öffentlichen Körperschaften (sogenannter öffentlicher Wald). Das heißt aber nicht, $\mathrm{da} ß$ für diese über $8000 \mathrm{~km}^{2}$ Wald Einheitlichkeit besteht in bezug auf die dem raumwirksamen Verhalten zugrundeliegende Wahrnehmung und Einschätzung seiner wirtschaftlichen, ökologischen und ideellen Bedeutung. Denn hinter dem Einheitsbegriff «öffentliche Körperschaften» stehen verschiedenste, in einem ganz bestimmten kulturellen Umfeld gewachsene Gemeinschaften wie Einwohner-, Bürger- oder Kirchgemeinden, Korporationen, Alp- oder Wuhrgenossenschaften, Pfrundstiftungen usw. (vgl. WULLSCHLEGER 1978). Ihnen steht als Grundeigentümer ein freier Entscheidungsraum für die Nutzung zu, der einerseits durch naturräumliche Bedingungen, andererseits durch gesetzliche $\mathrm{Be}$ stimmungen (FPolG, ZGB, kantonale Forstgesetze) eingegrenzt ist. Der Rahmen ist für den öffentlichen Wald enger gesteckt als für den Privatwald, indem für seine Pflege und Nutzung verbindliche Wirtschaftspläne erstellt und Hiebsätze festgelegt werden müssen. Auch wenn der Begriff «öffentlicher Wald» diesen Anschein erwecken könnte, ist es nicht die Allgemeinheit oder jeder Einzelne, der über dessen Nutzung entscheidet, sondern jeweils eine konkrete Körperschaft, gemäß deren Organigramm bestimmten Instanzen gewisse Entscheidungsbefugnisse zustehen. Die personelle Besetzung dieser Instanzen und deren kultureller Hintergrund spielen daher ebenfalls eine wesentliche Rolle. Ferner ist zu beachten, welchen Stellenwert der forstwirtschaftliche Teil im Gesamthaushalt des Eigentümers einnimmt. Während beispielsweise bei Korporationen die Holznutzung oft die Haupteinnahmequelle ist, hat derWald im Haushalt von Körperschaften mit eigener Steuerhoheit wie Einwohner- oder Kirchgemeinden eine andere Bedeutung.

Andreas Fischer, Dr., Bodenhofstr. 36, 6005 Luzern

Alois Kempf, Dr., Eidg. Anstalt für das forstliche

Versuchswesen, 8903 Birmensdorf 
Auch beim Wald vermag durch den Einbezug des unsichtbaren Ordnungsnetzes "Grundeigentum» das sichtbare Nutzungsmuster erhellt und interpretiert werden (GALLUSSER 1979: 155). Aus diesen Überlegungen wurde im Rahmen einer thematisch weit gefaßten Befragung 1983/84 in den Kantonen Luzern und Wallis der Gesichtspunkt der Einschätzung von Waldfunktionen durch die Eigentümer angesprochen. Die Gesamtergebnisse sind in FISCHER (1985) und KEMPF (1985) publiziert. Der vorliegende Beitrag beschränkt sich darauf, die Aussagen von 19 bzw. 34 Vertretern öffentlichen Waldeigentums aus den beiden Gebieten einander gegenüberzustellen und interregionale Gemeinsamkeiten oder Unterschiede zu erläutern. An anderem Ort (FISCHER 1985) wurde bereits ausgeführt, wie innerhalb eines Gebietes die Eigentümerkategorien Privat- und öffentlicher Wald weiter zu differenzieren wären.

$\mathrm{Da}$ eine Fokussierung auf die Waldeigentümer lohnt, kann beispielsweise der gesamtschweizerischen Umfrage von 1978 über die Einstellung der Bevölkerung zu Problemen des Waldes und der Waldwirtschaft (HERTIG 1979) entnommen werden, wo sich bei mehreren Fragen signifikant unterschiedliche Antworten für Waldeigentümer ergeben (z. B. bezüglich Waldfläche, Wildschäden, Walderschließung). Andererseits hatte dieselbe Befragung deutlich gemacht, wie schlecht die Bevölkerung im allgemeinen über die wirklichen Besitzverhältnisse beim Wald sowie über die Aufgaben und Pflichten derWaldbesitzer Bescheid wußte. Seit kurzem hat die Entschädigungsfrage infolge des Waldsterbens die Waldbesitzer erneut in die Diskussion gebracht (BOSSHARD 1984).

\section{Methode}

Grundlage der Erhebung sind längere, strukturierte Interviews, in welchen die hier ausgewerteten zwei Fragen integriert waren. Das Interview aber ist, gerade wenn es gilt, raumrelevante Verhaltensweisen $\mathrm{zu}$ erfassen, eine nur begrenzt adäquate Forschungsmethode. Auch bei unserer Untersuchung trat deutlich zutage, da $\beta$ damit zwar Einstellungen, Meinungen und sachliche Auskünfte zu erhalten sind. Ob die auf einer abstrakten Ebene formulierten Absichten jedoch ein entsprechendes Verhalten nach sich ziehen, kann höchstens angenommen werden. Es gälte, hier ein Instrumentarium zu entwikkeln, welches über das tatsächliche Verhalten besser Aufschluß geben könnte, ohne gerade die aufwendige teilnehmende Beobachtung anwenden zu müssen.

Wenn es sich, wie im vorliegenden Fall, bei den zu befragenden Aktoren um juristische Personen handelt, besteht eine zusätzliche Schwierigkeit darin, zu bestimmen, wer befragt werden soll. Als Interviewpartner kommt ja nur eine natürliche Person in
Frage. In bezug auf Waldnutzung sind bei Körperschaften meist mehrere Personen an raumrelevanten Entscheiden beteiligt. Je nach Organisationsgrad können dies das Forstpersonal, Spezialkommissionen, die Exekutive, die Legislative oder die stimmberechtigten Bürger bzw. Einwohner sein. Wir haben uns entschieden, nicht das Forstpersonal, das als Fachleute mit der Ausführung betraut wird, sondern einen politischen Vertreter des Eigentümers (Waldpräsident, Liegenschaftsverwalter) als Interviewpartner zu wählen. Es ist dabei zu berücksichtigen, daß oft auch Entscheide, die über forstgesetzliche Zuständigkeiten hinausgehen, den Kreisoder Revierförstern überlassen werden.

\section{Folgende zwei Fragen wurden den Befragten vorge-} legt:

1. Bewerten Sie bitte die Bedeutung IhresWaldes zu folgenden Punkten: a) Eigenbedarf an Holz, b) Einkommensquelle, c) Reserve für Notzeiten, d) Arbeitsmöglichkeit, e) Vermögensanlage, f) Schutzaufgaben, g) Erholungsraum (Skala vgl. Fig. 3).

Ausgangspunkt war die klassische Dreiteilung der Waldfunktionen in Nutz-, Schutz- und Erholungsfunktion, wobei wir die Produktivfunktion weiter differenzierten. Diese vorgegebenen Antwortkategorien mögen für einen groben Überblick genügen, für eine feinere Analyse, die sich nur mit Waldfunktionen befaßt, müßten die Kategorien genauer definiert und präziser abgegrenzt werden. So sind vor allem «Vermögensanlage» und «Reserve für Notzeit» noch zu stark miteinander verflochten, was auch für «Arbeitsmöglichkeit» und «Einkommensquelle» gilt. Ebenso müßten Schutz- und Erholungsfunktionen so weit aufgefächert werden, daß ersichtlich wird, ob die Wirkung für den Eigentümer oder diejenige für die Allgemeinheit beurteilt wird. Anzumerken ist, daß es sich bei der Zuordnung von Waldfunktionen nicht um Aufgaben und Leistungen einzelner Waldungen an und für sich handelt. Vielmehr umschreiben Waldfunktionen Zuweisungen von Interessen bestimmter sozialer Gruppen.

2. Bitte geben Sie für die folgenden Faktoren an, welche Rolle sie bei der Bestimmung der jährlichen Holznutzung spielen: a) Löhne derWaldarbeiter, b) Anfall von Schadholz, c) Holzpreis, d) Waldbauliche Notwendigkeit, e) Eigenbedarf an $\mathrm{Holz}$, f) Betriebliche Notwendigkeit, g) Hiebsatz des Wirtschaftsplans, h) Finanzielle Notwendigkeit (Skala vgl. Fig. 4).

In der Forstwirtschaft stellt die Holznutzung ein sehr wichtiges raumrelevantes Verhalten dar. Die Nutzungsmenge ist dabei ein Element neben anderen, wie z. B. Baumartenselektion, Hiebsart oder Hiebsort. Nach den Bestimmungsfaktoren für die Nutzungsmenge wurde gefragt, weil wir von der Hypothese ausgingen, daß sie sich in der Gewichtung regional und nach Eigentümerkategorien unterscheiden. Waldbauliche Überlegungen, insbeson- 
dere das Gebot der Nachhaltigkeit, beeinflussen die Höhe der Nutzungsmenge. Der Hiebsatz im Wirtschaftsplan als maximale Nutzungsmenge innerhalb eines bestimmten Zeitraumes (mittelfristig) berechnet sich nach diesem Gebot. Weitere Rahmenbedingungen für den Eigentümer sind die Lohnkosten der Waldarbeiter und der Holzpreis. Vom Eigentümer steuerbare Bestimmungsgrößen sind hingegen der Eigenbedarf an Holz, die betriebliche Notwendigkeit (Auslastung von festangestelltem Personal, Amortisation von Maschinen und Einrichtungen) und die finanzielle Notwendigkeit (Kapitalbedarf für forstliche Investitionen oder anderweitige Vorhaben wie Kirchenbau, Straßenunterhalt).

\section{Vergleichsgebiete Kanton Luzern und Oberwallis}

Im Kanton Luzern handelt es sich bei den 19 befragten Waldeigentümern um Einwohner-, Bürger-,
Kirchgemeinden und kirchliche Stiftungen, Realund Personalkorporationen, den Bund und den Kanton Luzern mit Waldeigentum in den Gemeinden Schwarzenberg, Malters, Neuenkirch, Sempach und Neudorf. In diesen Gemeinden gehören aber $67 \%$ der Waldfläche 771 privaten Eigentümern, die durchschnittlich etwa 2 ha Wald besitzen. Wenn für den regionalen Vergleich Luzern-Oberwallis diese verschiedenen öffentlichen Waldeigentümer in eine Kategorie zusammengefaßt werden, so ist festzuhalten, daß zwischen den einzelnen Typen von öffentlichen Waldeigentümern bei den Antworten beträchtliche Differenzen auftreten können. Für eine feinere Analyse ist deshalb die Zusammensetzung dieser heterogenen Gruppe und ihre räumliche Manifestation von Bedeutung. Um die heutige räumliche Verteilung der einzelnen Waldeigentümertypen und deren Ansprüche an den Wald verständlich zu machen, ist eine vertiefte Beschäftigung mit der Waldeigentumsentwicklung nötig. So

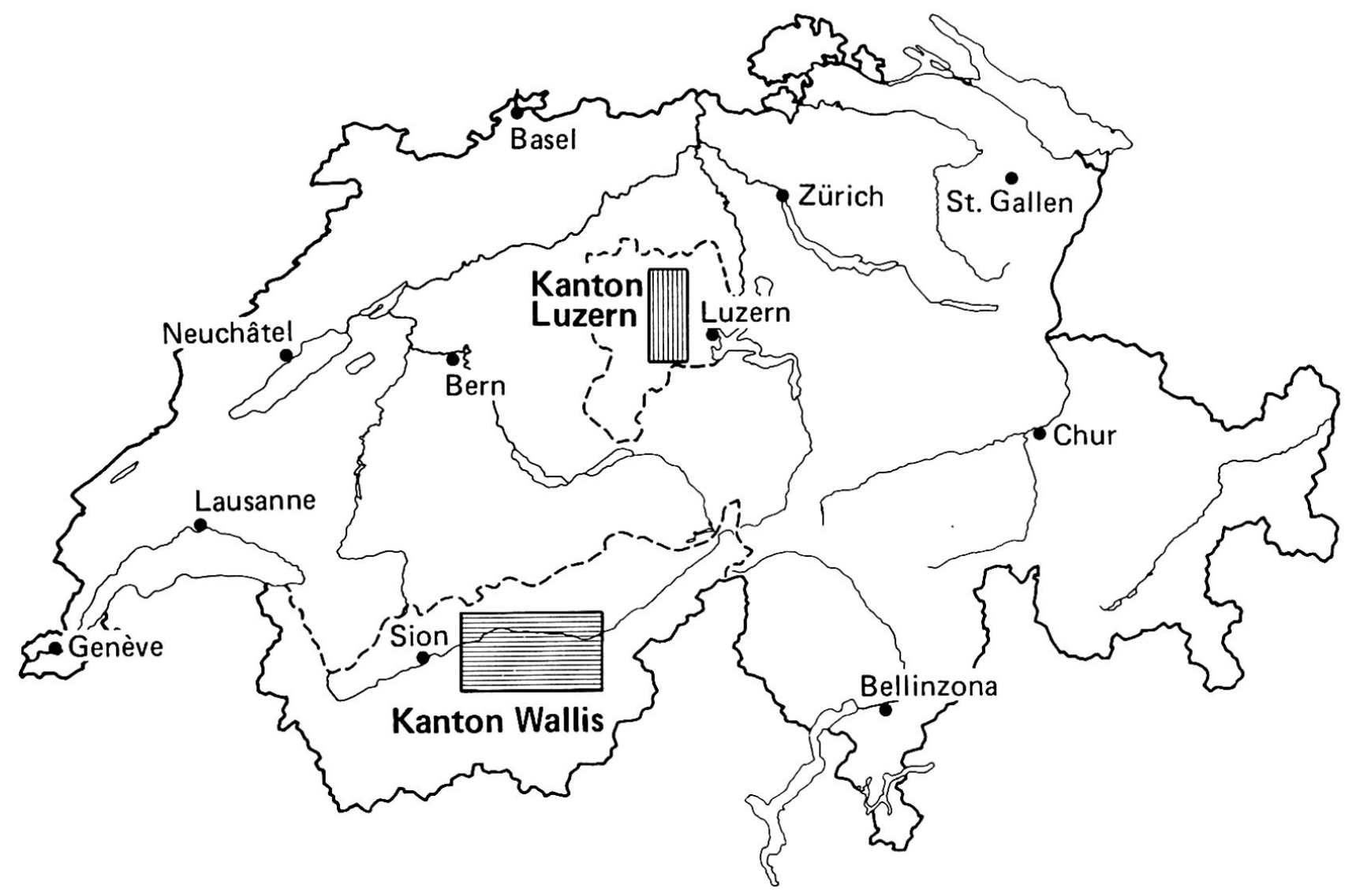

Luzerner Untersuchungsgemeinden

Untersuchungsgemeinden Oberwallis

Fig. 1 Untersuchungsgebiete 
finden als wichtige Aspekte in der bewegten Geschichte des Grundeigentums alte Waldreglemente und Erlasse aufgrund von Streitigkeiten, die verbreiteten Waldteilungen zu Beginn des 19. Jahrhunderts, die auch eigentumspolitisch bedeutsame Forstgesetzgebung ab 1875 und die Waldzusammenlegungen der neueren Zeit einen nicht nur im Grundbuch, sondern oft auch im Nutzungsmuster sichtbaren, räumlichen Niederschlag. Wie am Beispiel von Neudorf/LU gezeigt wird (vgl. Fig. 2), hat sich die heutige, vielfältige Eigentümerstruktur mit 5 öffentlichen und 108 privaten Waldeigentümern im Laufe von über 600 Jahren in mehreren Etappen aus dem alleinigen Eigentum des Stiftes Beromün- ster herausgebildet. Ebenso interessante und fein differenzierte Entwicklungen findet man im Luzerner Voralpengebiet, wo in der räumlichen Betrachtung zusätzlich die Höhenstufung Bedeutung erlangt. Für den kulturlandschaftlichen Wandel, aber auch für den Stellenwert des öffentlichen Waldes im Kanton Luzern sind Fragen der Eigentümerstruktur und ihrer räumlichen Entwicklung als Grundlage ebenso wichtig wie die nachfolgenden naturräumlichen und forstwirtschaftlichen Charakterisierungen. So sind beispielsweise die Ansprüche, insbesondere der ländlichen Bevölkerung, an den öffentlichen Wald geprägt durch die breite Streuung des Waldeigentums (rund 12000 private Waldeigentü-

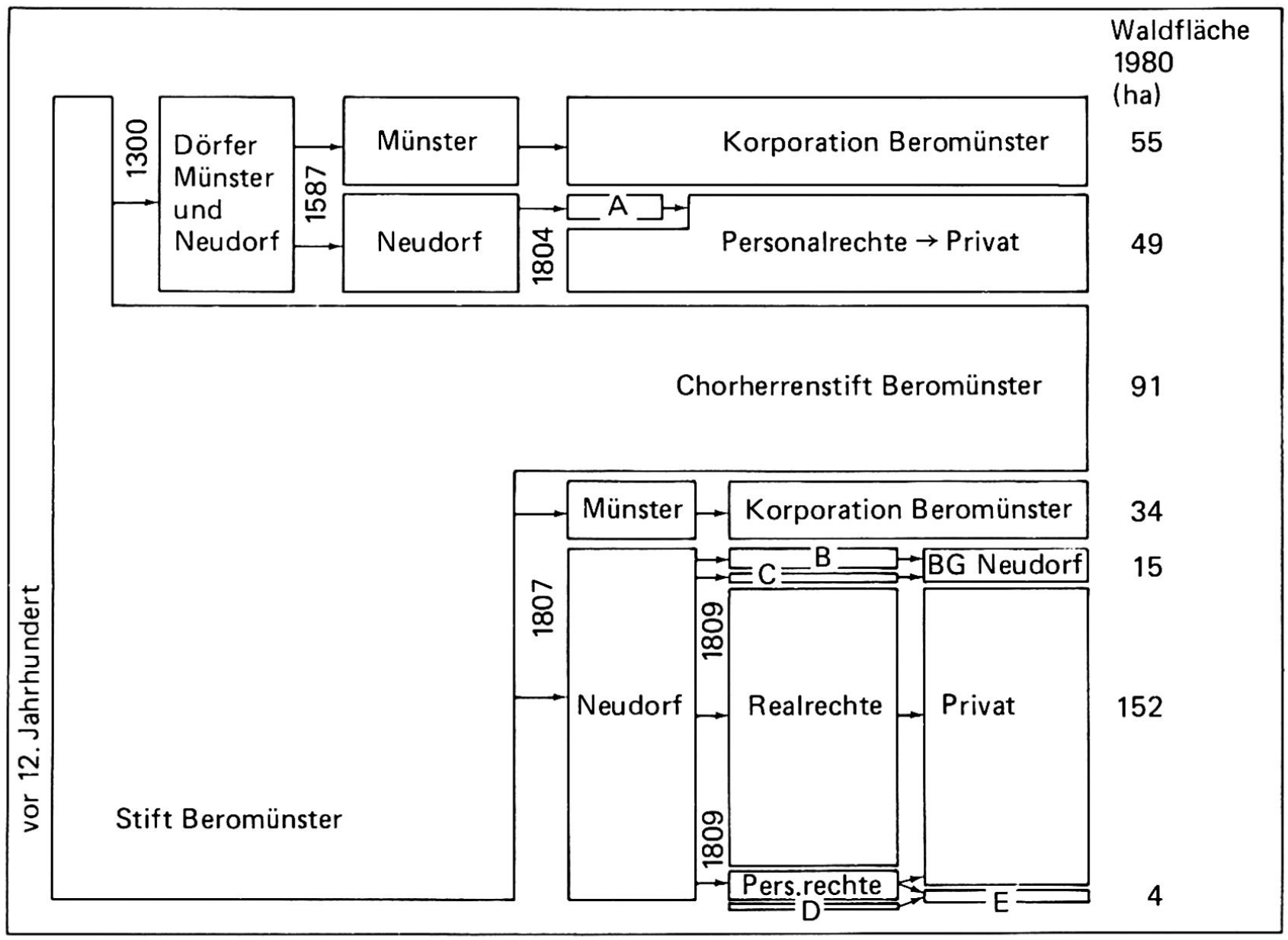
A Reservewald
B Pfarrpfrund und Gormunderpfründe
C Waisenanstalt
D Aufforstung von Korporationsland Neudorf
E Korporation Neudorf

$B G=$ Bürgergemeinde

Fig. 2 Schema der Waldeigentumsentwicklung in der Gemeinde Neudorf/LU 
mer) und die traditionell enge Verknüpfung zwischen Land- und Forstwirtschaft. Übrigens sind 11 der 19 befragten Vertreter öffentlichen Waldes Privatwaldeigentümer.

Der obgenannte Untersuchungsraum im Kanton Luzern läßt sich kurz wie folgt charakterisieren: er stellt einen Ausschnitt aus dem Übergangssaum Luzerner Mittelland-Voralpen dar, der klimatisch zu den Regionen Alpennordhang und zentrales Mittelland, mit ausgeprägter Gewitter- und Hagelhäufigkeit zu zählen ist. Die unteren Lagen werden bis etwa $1000 \mathrm{~m}$ ü. M. vom Buchen-Tannengürtel eingenommen, während sich darüber bis zur Waldgrenze der Fichtengürtel erstreckt, der in höheren Lagen von baumartigen Bergföhren und Grünerlengebüsch abgelöst wird. Die Bewaldung beträgt $29 \%$ und schwankt zwischen $8 \%$ und $47 \%$. Die folgenden forstlichen Kennzahlen betreffen den ganzen Kanton Luzern: Nadelholzanteil $83 \%$; geschätzter jährlicher Holzzuwachs 7,4 m³/ha; Holzabgabe 1984 $4,4 \mathrm{~m}^{3} / \mathrm{ha}$ (öffentlicher Wald 7,3 $\mathrm{m}^{3} / \mathrm{ha}$ ); mehrheitlich Stammholznutzung (1984 71\%); Eigenbedarf an Holz beim öffentlichen Wald 6,8\%. Ein Drittel des Luzerner Waldes ist, vor allem im nördlichen Kantonsteil, zusammenlegungsbedürftig. Für $60 \%$ dieser Fläche sind bis 1986Waldzusammenlegungen beschlossen, in Bearbeitung oder bereits vollendet worden. Waldzusammenlegungen sind ein markantes Ereignis im Wandel der Waldbewirtschaftung (Nutzung und Pflege), weil die Parzellar- und teilweise auch die Eigentümerstruktur sowie die Walderschließung völlig umgestaltet werden.

Im Oberwallis wurden alle Bürgergemeinden des Rhonetales zwischen Brig und Salgesch (Pfynwald) befragt. Sie verfügen nach Schätzungen etwa über $85-90 \%$ der Waldfläche dieser Gegend. In den Gemeinden Brig, Eggerberg, Hohtenn, Inden, Leuk, Salgesch und Visp führten 1983 die Bürgergemeinden jeweils eine von der Einwohnergemeinde getrennte Verwaltung mit eigenem Bürgerrat. Der Staat Wallis und die Einwohnergemeinden spielen als Waldeigentümer keine Rolle. Die schweizerische Eidgenossenschaft besitzt Wald in der Umgebung von militärischen Anlagen. Zweitwichtigster Eigentümer öffentlichen Waldes sind hier die Alpgenossenschaften (Geteilschaften). Zum Privatwald, der in ein paar Gemeinden über 15\% derWaldfläche ausmacht, liegen bisher kaum statistische und raumbezogene Angaben vor. Einzelheiten zur Befragung und zum Waldeigentum in der Region findet man in KEMPF (1985: 24-27, 35-39, 101-128). Neben dem Hauptmerkmal «Bürgergemeinde- und Genossenschaftswald» mögen folgende Charakteristika für eine forstliche Kennzeichnung genügen: zentralalpine Vegetation; überwiegend Nadelwald mit Fichten, Tannen, Föhren, Lärchen und Arven; Bewaldungsprozent in den Gemeinden zwischen $10 \%$ und $60 \%$; Holzzuwachs ca. $1,1 \mathrm{~m}^{3} / \mathrm{ha} / \mathrm{Jahr}$; Holznutzung unter $1 \mathrm{~m}^{3}$; mehrheitlich Stamm- und Industrieholz zum Verkauf; Eigenbedarf bei etwa $10 \%$. Die Betriebsstrukturen sind durch den Aufbau von interkommunalen Forstrevieren mit ständigem Forstpersonal und einer verbesserten Erschließung der Gebirgswälder in den letzten Jahren einer starken Veränderung unterworfen. Die befragten Bürgergemeinden müssen in der Mehrzahl ohne einen aktuellen Waldwirtschaftsplan auskommen.

\section{Antworten zur Bedeutung des Waldes}

Vergleicht man die Antworten der Befragten zur allgemeinen Bedeutung «ihres» öffentlichen Waldes (Figur 3), so fällt die Ähnlichkeit in den beiden Regionen auf. Eine Ausnahme bildet der Aspekt «Eigenbedarf an Holz», den im Oberwallis $76 \%$ der Gesprächspartner als wichtig einstufen, gegenüber $15 \%$ im Raume Luzern. Im übrigen ergibt sich eine gemeinsame Hierarchie: die höchsten Nennungen für Erholung, Schutzaufgaben und Reservefunktion, im Mittelfeld die finanziellen Gesichtspunkte und am Schluß jene der Beschäftigung.

Eigenbedarf an Holz

Einkommensquelle

Reserve für Notzeit

Arbeitsmöglichkeit

Vermögensanlage

Schutzaufgaben

Erholungsgebiet
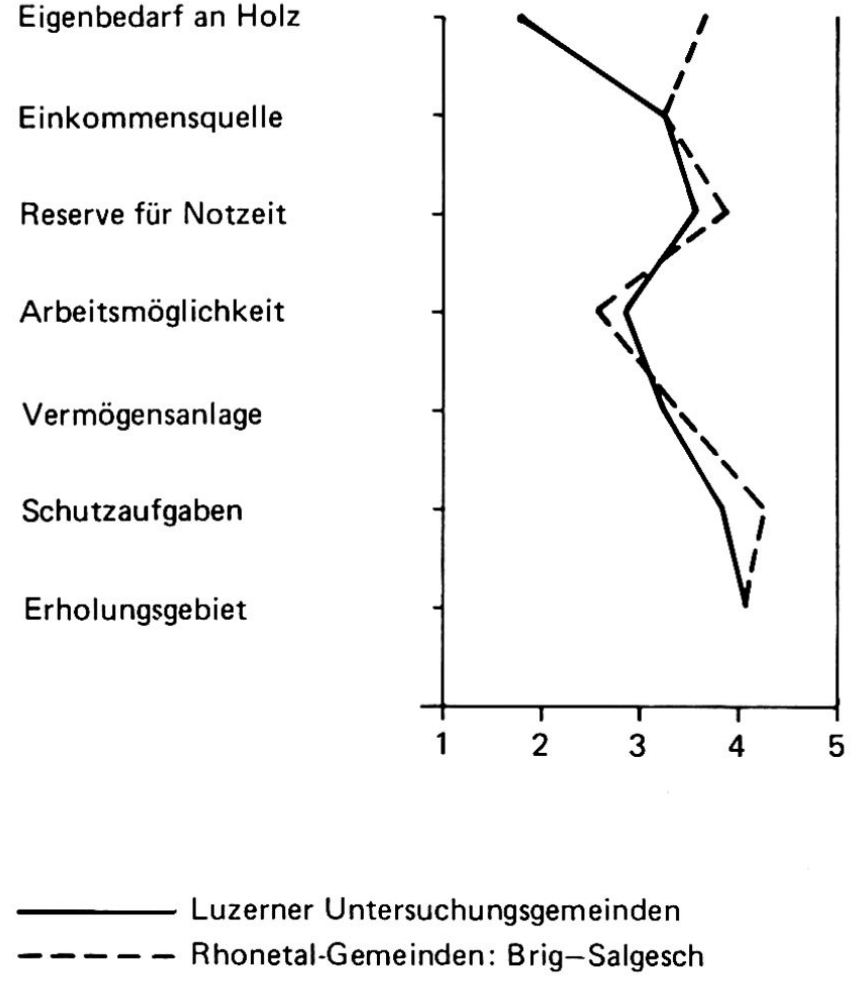

Skala: $\quad 1=$ sehr unbedeu tend $2=$ unbedeutend

$4=$ wichtig $3=$ unentschieden

$5=$ sehr wichtig

Fig. 3 Bedeutung einzelner Waldfunktionen 
Die Unterschiede in der Rangfolge Schutz-/Erholungsfunktion lassen sich räumlich erklären: reliefbedingt erreicht die protektive Funktion des Walliser Gebirgswaldes, der Siedlungen und Verkehrswege im Rhonetal schützen muß, einen höheren Stellenwert als im Kanton Luzern, wogegen diese Art von Schutz in den Mittellandgebieten von geringerer Bedeutung ist. Die primäre Schutzbedeutung des Gebirgswaldes wurde ebenfalls bestätigt durch Aussagen von 9 Vertretern der Alpgenossenschaften im Raume Simplon-Nord und Nanztal zur selben Frage. Der Erholungswert desWaldes wird in den beiden Gebieten gleich hoch eingeschätzt. Damit steht der Erholungsaspekt im Kanton Luzern im ersten Rang. Diese Rangierung entspricht in der Tendenz den Ergebnissen der Umfrage von 1978, wo die Schutz- und Erholungsfunktionen an erster Stelle stehen, während der Wald als Wirtschaftsfaktor bedeutend geringer eingeschätzt wurde (HERTIG 1979).

Ausgeprägter ist die Differenz beim Eigenbedarf. Die folgenden Ausführungen mögen seine geringe Bedeutung im Kanton Luzern erklären. Charakteristisch für die Luzerner Eigentumsverhältnisse ist der mit $70 \%$ der Fläche ausgesprochen hohe Privatwaldanteil. Beim öffentlichen Wald stellen die Korporationsgemeinden die wichtigste Eigentümergruppe dar (knapp 50\% des öffentlichen Waldes). Die Nutznießer (Korporationsbürger) besitzen, sofern sie Landwirte sind, meist selbst noch eigenen Wald, aus dem sie den Eigenbedarf an Holz decken. Die Nichtlandwirte haben, im Zeitalter der Öl- und Elektroheizungen, meist keinen Bedarf mehr an Holz. Die früher übliche Entrichtung des Bürgernutzens in Form von Brennholz wird heute, sofern es die finanzielle Lage erlaubt, meist in bar oder mit einem Essen/Ausflug abgegolten. Der Eigenbedarf für den Betrieb von Bürgerheimen, für die Erstellung von Bauten oder für die eigenen Gutsbetriebe ist relativ gering. Es kommt sogar vor, da $\beta$ aus betrieblichen und wirtschaftlichen Gründen der gesamte Holzschlag verkauft wird und ein eventueller Eigenbedarf an Holz entsprechend zugerüstet angekauft wird.

Diese Angaben gelten im großen und ganzen analog auch für die übrigen öffentlichen Waldeigentümer, die sich damit ganz wesentlich von den Privatwaldeigentümern, im speziellen den Landwirten, unterscheiden. Bei ihnen steht der Eigenbedarf in der Bedeutung klar an erster Stelle, während Erholungsund Schutzfunktionen erst im dritten und vierten Rang folgen. Die durchschnittliche Bewertung des Eigenbedarfs beträgt durch die Landwirte 4,6 Punkte, durch die Privatwaldeigentümer insgesamt 4,3 Punkte, durch die öffentlichen Waldeigentümer aber nur 1,8 Punkte (vgl. Fig. 3 und FISCHER 1985: 59).

Die Einschätzung im Walliser Rhonetal ist auf einem anderen Hintergrund zu sehen. 1983 gaben
$65 \%$ der Informanten an, daß die Bürgergemeinde auf Anfrage noch Losholz (1-5 Ster Brennholz) abgäbe, und gar $80 \%$ der 34 Burgerschaften kannten einen Holznutzen für Bauholz (in der Regel $10-50 \mathrm{~m}^{3}$ ). Eine Hemmschwelle für den Bezug des forstlichen Bürgernutzens bildete der vom Nutzungsberechtigten oft selbst zu leistende Arbeitsaufwand. Dennoch war zum Zeitpunkt der Umfrage die Zahl der Losholz beziehenden Personen im Steigen begriffen. Es war bei der Bewertung kein deutlicher Unterschied zwischen Berg- und Talgemeinden auszumachen. Insgesamt hat die Rolle des Waldes zur Deckung des eigenen lokalen Holzbedarfs seine frühere Wichtigkeit auch im Walliser Untersuchungsgebiet eingebüßt.

\section{Antworten zur Holznutzung}

Die Ergebnisse in den beiden Gebieten zeigen bei der Frage, welche Faktoren für die jährliche Holznutzung wichtig sind, insgesamt mehr Abweichungen (vgl. Fig. 4). Größere Differenzen bestehen beim Hiebsatz und der waldbaulichen Notwendigkeit, wo im Luzernischen höhere Wertungen fallen als im Oberwallis, sowie beim Eigenbedarf und beim Kapitalbedarf in umgekehrter Reihenfolge. Während in den Luzerner Untersuchungsgemeinden waldbauliche Notwendigkeit und Hiebsatz vor dem Holzpreis an der Spitze stehen, führt im Oberwallis der Holzpreis die Rangfolge an, und der Hiebsatz steht in der Bedeutung an zweitletzter Stelle. Beiden Gebieten gemeinsam ist die geringe Bedeutung der betrieblichen Notwendigkeit und der Löhne für die Waldarbeiter.

Für die Interpretation ist vorauszuschicken, da $ß$ der Hiebsatz eigentlich die Nutzungsmenge aufgrund der Ergebnisse des Waldwirtschaftsplans verbindlich festsetzt. Der Hiebsatz orientiert sich am waldbaulichen Prinzip der Nachhaltigkeit. Hiebsatz und "waldbauliche Notwendigkeit» sind insofern verknüpft, als der Hiebsatz ein längerfristiges Planma $\beta$ darstellt, die waldbauliche Notwendigkeit sich aber eher auf die aktuelle Situation bezieht. Daß diese beiden Größen im Kanton Luzern hoch bewertet werden (1. und 2. Rang), läßt sich auf folgendes zurückführen: fast der gesamte öffentliche Wald ist, meist seit Jahrzehnten, eingerichtet, das heißt, waldbauliches Grundlagenmaterial in Form von Waldwirtschaftsplänen steht zur Verfügung. Das Kreisforstamt übt zudem über die Anzeichnung der zu schlagenden Bäume eine gewisse Kontrollfunktion aus. Größere öffentliche Waldeigentümer beschäftigen eigenes Fachpersonal (diplomierte Förster). In dieser Bewertung spiegeln sich somit mehr als hundert Jahre kantonales Forstgesetz mit den in der Folge durchgesetzten Verbesserungen der Waldbewirtschaftung durch Professionalisierung der Be- 


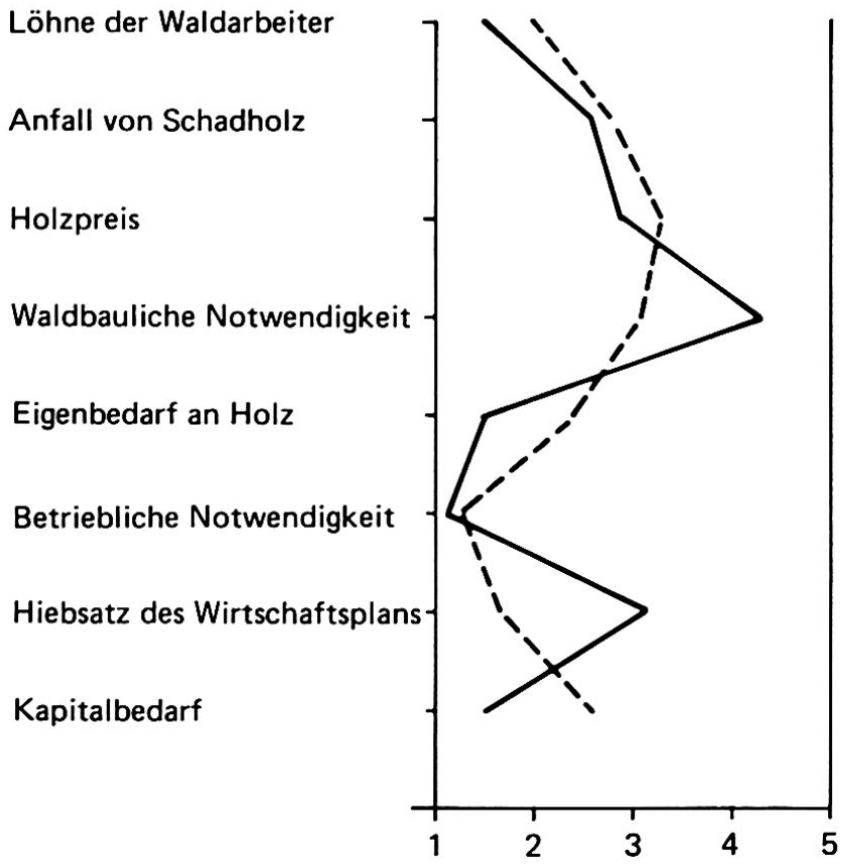

\begin{tabular}{lll} 
& Luzerner Untersuchungsgemeinden \\
\hline$-1-$ & & \\
Skala: & $1=$ Rhonetal-Gemeinden: Brig-Salgesch \\
& $2=$ wenig Einfluß & $4=$ starker Einfluß \\
3 & $=$ mittlerer Einfluß &
\end{tabular}

Fig. 4 Beurteilung von Faktoren bei der jährlichen Holznutzung

försterung und Einführung der Waldwirtschaftspläne. Wenn auch der Holzpreis bereits an dritter Stelle der Bestimmungsfaktoren für die Holznutzung steht, so wird doch klar, daß kurzfristige ökonomische Vorteile zurückstehen vor dem prioritären Gebot der Nachhaltigkeit, was übrigens in Übereinstimmung steht mit der hohen Bewertung der Erholungs- und Schutzfunktionen.

Die Wertungen im Oberwallis lassen sich gut mit der bis vor kurzem vorherrschenden forstbetrieblichen Situation in Beziehung bringen. Eine betriebliche Notwendigkeit bestand meist nicht, da die Bürgergemeinden zwar einen Forstpolizisten angestellt hatten, nicht aber einen eigenen Forstbetrieb unterhielten. Drei Viertel der befragten Burgerschaften verfügten 1983 nicht über eigene Einsatzmittel wie Motorsäge, Seilanlage oder Forstfahrzeuge, und ein ähnlich hoher Prozentsatz ließ die Holzernte durch ein mit Maschinen und Geräten ausgestattetes Holzerunternehmen ausführen. Der Hiebsatz spielt wohl auch deshalb eine geringe Rolle, weil nur zu wenigen Bürgerwaldungen einschlägige In- formationen aus aktuellen Wirtschaftsplänen vorliegen. Solche betrieblich-organisatorische Rahmenbedingungen erlauben bei der Holznutzung ausser der waldbaulichen Notwendigkeit (inkl. Schadholz) die Entwicklung des Holzpreises stärker mitzuberücksichtigen.

Eine zweite Differenz besteht in der unterschiedlichen Bewertung der Rolle des Kapitalbedarfs für die Bestimmung der Nutzungsmenge. Obwohl im Kanton Luzern die Reservefunktion des Waldes relativ hoch geschätzt wurde (vgl. Fig. 2), wird die Holznutzung praktisch nicht durch den Bedarf des Eigentümers an finanziellen Mitteln bestimmt. Es wurde oben bereits dargelegt, da $\beta$ ökonomische Überlegungen eine sekundäre Rolle spielen. Dabei muß zusätzlich berücksichtigt werden, $\mathrm{da} \beta$ die $\mathrm{Be}$ fragung zu einem Zeitpunkt mit einem Überangebot an Holz auf dem Markt und deshalb tiefen Holzpreisen, andererseits aber hohen Waldarbeiterlöhnen stattfand. Die meisten Waldrechnungen schlossen knapp ausgeglichen, und große Erträge aus erhöhter Nutzung schienen in naher Vergangenheit und Zukunft nicht realisierbar. Warum der finanzielle Gesichtspunkt im Wallis gegenüber den öffentlichen Waldbesitzern im Kanton Luzern mehr Betonung findet, ist nicht direkt ersichtlich. Auch dort haben sich die Bürgergemeinden an sozialen Aufgaben in den Gemeinden zu beteiligen, und mancher Holzschlag dient dem Unterhalt eigener Gebäude. Die Löhne der Waldarbeiter sind im Oberwallis von geringem Einfluß. Ohne betriebliche Infrastruktur entstanden gewöhnlich vor allem dann Kosten, wenn auch Einnahmen aus Holzschlägen zu verzeichnen waren. In beiden Gebieten war übrigens die Bedeutung des Waldes für die Beschäftigung (Arbeitsmöglichekit) an letzter Stelle als eher unbedeutend zu finden.

\section{Folgerungen}

Die beschriebenen Fragen zur Bedeutung einzelner Waldfunktionen und zu Begründungen für die jährliche Holznutzung aus der Sicht öffentlicher Waldeigentümer sind ein recht bescheidenes «Meßinstrument», wie im methodischen Abschnitt bereits angetönt wurde. Wichtige Ansätze zum Aufdecken von kulturräumlichen Zusammenhängen und landschaftsrelevanten Entscheidungsprozessen aller am Walde interessierten Gruppen werden von seiten der Geographie auch im Rahmen der Implementations- und Perzeptionsforschung eingebracht. In der Forstwissenschaft werden ähnliche Fragestellungen in forstpolitischen Studien angegangen (NIESSLEIN 1985, SCHAERER/ZIMMERMANN 1984). Die Aussage von WINDHORST (1978: 21), wonach es an Arbeiten zur Erfassung der forstlichen Aktivitäten von sozialen Gruppen und ihrer räumlichen Verteilung besonders fehle, hat freilich nur wenig an Aktualität 
eingebüßt. Wir hoffen deshalb, mit vorliegendem Vergleich anzuregen, da $B$ Geographen stärker als bisher das Landschaftselement Wald in sozialgeographische Untersuchungen einbeziehen oder den Wald von einem integralen Ansatz aus direkt als Forschungsgegenstand wählen.

\section{Kurzfassung}

Die Aktivitäten sozialgeographischer Gruppen bilden einen wichtigen Themenbereich forstgeographischer Forschung. Ein Teilaspekt dazu sind Untersuchungen zum Grundeigentum. In der Schweiz gelangen die Waldbesitzer, verglichen mit dem Forstdienst und den Waldbenützern, eher selten zu Wort. Im Rahmen einer thematisch weiter gefaßten Studie wurde in der Umgebung von Luzern und im Oberwallis nach der Bedeutung des öffentlichen Waldes aus der Sicht der Waldeigentümer gefragt. Der interregionale Vergleich zeigte trotz der naturräumlichen Verschiedenheit viele Ähnlichkeiten und bestätigte die Gewichtungen aus einer gesamtschweizerischen Umfrage von 1978, wo die Schutzund Erholungsfunktionen des Waldes vor wirtschaftlichen Überlegungen rangierten. Besonders aufgefallen sind im Oberwallis die Bedeutung der Holznutzung zwecks Eigenbedarf und im Kanton Luzern die Rolle der waldbaulichen Planung. Nach Meinung der Verfasser sollte dem Eigentumsaspekt in der Forstpolitik größere Beachtung geschenkt werden, wozu freilich spezielle Untersuchungen, auch von Geographen, Grundlagen erarbeiten sollten.

\section{Summary}

On the evaluation of forest functions in different regions by representatives of publicly-owned forest. The activities of socio-geographic groups constitute an important field of research in forest geography. One aspect of such research involves the study of land ownership. In Switzerland, the views of the forest owners, as opposed to those of the forest service and forest users, are comparatively seldom considered. As part of a broader study, the importance of the public forest as seen by the forest owners was investigated in the neighbourhood of Lucerne and in the German-speaking part of the Valais. Despite the differences in geographical conditions, comparison of the two regions revealed many similarities, and confirmed the weightings established in the nationwide survey of 1978 , according to which the protective and recreational functions of the forest were ranked above economic considerations. Particular emphasis was laid on the importance of wood harvesting for home consumption in the Valais, and on the role of silvicultural planning in the canton of Lucerne. In the opinion of the authors, forest policies should pay more attention to the aspect of ownership. Geographers can participate in providing the necessary specialised information.

\section{Literaturnachweis}

BOSSHARD, W. (1984): Die schweizerischen Waldbesitzer vor der Bedrohung sterbender Wälder. In: Forst- und Holzwirt, Nr. 39, 203-205.

de COULON, M. (1983): Politique forestière et forêt privée. In: Schweizerische Zeitschrift für Forstwesen, Nr. 134, 703-712.

EWALD, K. C. (1978): Der Landschaftswandel. Zur Veränderung schweizerischer Kulturlandschaften im 20. Jahrhundert. In: Tätigkeitsberichte der Naturforschenden Gesellschaft Baselland, Nr. 30, 55-308.

FISCHER, A. (1985): Waldveränderungen als Kulturlandschaftswandel - Kanton Luzern. Basler Beiträge zur Geographie, Nr. 32.

GALLUSSER, W. A. (1979): Über die geographische Bedeutung des Grundeigentums. In: Geographica Helvetica, Nr. 34, 153-162.

HERTIG, H. P. (1979): Die Einstellung der Bevölkerung zu Problemen des Waldes und der Waldwirtschaft. In: Schweizerische Zeitschrift für Forstwesen, Nr. 130, 591-620.

KEMPF, A. (1985): Waldveränderungen als Kulturlandschaftswandel - Walliser Rhonetal. Basler Beiträge zur Geographie, Nr. 31.

NIESSLEIN, E. (1985): Forstpolitik. Ein Grundriß sektoraler Politik. Pareys Studientexte, Nr. 47, Hamburg.

SCHAERER, W.; ZIMMERMANN, W. (1984): Politische und rechtliche Betrachtungen zum Thema Waldsterben in der Schweiz: eine Standortsbestimmung. Beiheft zur Schweizerischen Zeitschrift für Forstwesen, Nr. 73.

WINDHORST, H. W. (1978): Geographie der Wald- und Forstwirtschaft. Teubner Studienbücher Geographie. Stuttgart.

WULLSCHLEGER, E. (1978): Die Entwicklung und Gliederung der Eigentums- und Nutzungsverhältnisse am Wald. Eidgenössische Anstalt für das forstliche Versuchswesen, Berichte, Nr. 183. 